\title{
Reforma sanitaria, equidad y derecho a la salud en Colombia
}

\author{
Health reform, equity, and the right \\ to health in Colombia
}

Mario Hernández 1

\footnotetext{
1 Departamento de Salud Pública y Tropical, Facultad de Medicina, Universidad Nacional de Colombia. Ciudad Universitaria, Carrera 30 Calle 45, Bogotá D.C., Colombia. mha062@cable.net.co
}

Abstract The author devel ops a long-term perspectiveto assess advances in equity and the right to health in the Colombian health system reform. In a restricted political system, actors in the field of health in Colombia have chosen individualistic al ternatives to legal ize inequities in individual purchasing power for services. Despite the complex regulations establi shed in the General System for Social Security in Health, there is a trend towards consolidating traditional inequiti es and to further restrict opportunities for achieving the right to health with full, equitable, universal guarantees.

Key words Equity; Heal th Care Reform; Health Rights; Health System

Resumen Se presenta una visión delargo plazo para la valoración de los avances en la equidad y en el derecho a la salud de la reforma del sistema de salud en Colombia. En medio de un sistema político restringi do, los actores del campo dela salud en Colombia han construido opciones de tipo individual ista que ti enden a legal izar las desi gualdades ligadas a la capacidad de pago de las personas. A pesar de los complejos mecanismos de regulación estableci dos en el nuevo Sistema General de Seguridad Social en Salud, Ia tendencia apunta hacia una consolidación de las mismas desigualdades tradicionales y al distanciamiento de una garantía plena, equitativa y universal del derecho a la salud.

Palabras clave Equidad; Reforma en Atención de la Salud; Derecho a la Salud; Sistema de Salud 
Colombia adelanta una reforma profunda de su sistema de salud desde 1994 que ha sido promovida internacionalmente como un modelo aplicable para los países de América Latina (Frenk \& Londoño, 1997). Por medio de la Ley 100 de 1993 (Colombia, 1993), se pasó de un Sistema Nacional de Salud (SNS) a un Sistema General de Seguridad Social en Salud (SGSSS). El modelo ha resultado atrayente porque incorpora elementos centrales de la lógica del mercado de servicios en todo el sistema, como la competencia y la libre elección del usuario, al tiempo que desarrolla mecanismos de regulación, de solidaridad financiera entre ricos y pobres, y nuevas funciones de la función pública estatal. Según la Organización Mundial de la Salud (OMS, 2000), el hecho de que, en Colombia, los ricos gasten mucho más que los pobres explica su primer lugar en el mundo en el indicador de "equidad financiera". No obstante, esta perspectiva de evaluación tiende a ocultar los problemas de equidad de la reforma que apenas comienzan a ponerse en evidencia después de seis años de implementación del modelo (Agudelo, 1999; Céspedes et al., 2000; Eslava, 1999; Hernández, 2000a; Málaga et al., 2000; Restrepo, 2000).

El debate se ha movido entre los alcances del modelo mismo y los problemas de su aplicación. Pero los modelos de organización de los sistemas de salud no son simplemente conocimientos aplicados. Son el resultado de un proceso sociopolítico que realizan cotidianamente las fuerzas organizadas de la sociedad particular, en medio de las tensiones entre la dinámica internacional, la nacional y la local, entre lo estructural y lo coyuntural, lo que cambia y lo que se resiste (Hernández, 2000c). En este artículo, trataré de presentar una síntesis del proceso sociopolítico que conduce del SNS al de SGSSS en Colombia, para tratar de ubicar en tal proceso los problemas de equidad en los dos sistemas y los alcances y limitaciones de la reforma en curso.

El sistema nacional de salud: la fragmentación propia del mercado

Colombia, como todos los países latinoamericanos, ha sido afectada por las tendencias mundiales de la consolidación de los Estados nacionales y de la formación de los sistemas de salud durante todo el siglo XX. Así, la ausencia de opciones técnicas no explica la falta de un sistema más universalista de servicios de salud en Colombia. Esto se explica por el tipo de relaciones de poder en el sistema político, en general, y del campo de la salud y de la seguridad social, en particular. El sistema de salud, organizado jurídicamente en la década del 70, fue el resultado de tensiones y negociaciones complejas entre diferentes actores sociopolíticos de la sociedad colombiana, que han obtenido sólo parcialmente lo que cada uno pretendía. Aún así, grandes sectores de la población han quedado excluidos, y se ha acumulado una enorme frustración en medio de los intentos fallidos por ofrecer, por lo menos, servicios de atención médica para todos (Hernández, 2000a).

De tiempo atrás, la sociedad colombiana venía construyendo diferentes formas de atención de los problemas de salud. Hacia la década del 60, había cinco formas diferentes de atención: (1) los servicios para los ricos o "pudientes", ofrecidos por el ejercicio privado de la profesión médica en consultorios y clínicas, a veces apoyado en algún seguro privado; (2) el seguro obligatorio, en sus múltiples formas, que atendía a los trabajadores formales o asalariados de los sectores privado y público; (3) la atención de los pobres, mediante dos estrategias basadas en la caridad, ya fuera pública bajo el nombre de "asistencia pública”, o privada bajo el nombre de "beneficencia"; (4) los mecanismos de atención y control de epidemias y enfermedades de alto impacto colectivo, denominados de "salud pública" y a cargo del Estado; y (5) todas las prácticas médicas populares no científicas, producto del sincretismo cultural acumulado, pero ubicadas en un lugar marginal y siempre subvalorado por los servicios legalmente aceptados. Así, los servicios de saIud constituían una organización social fragmentada con base en la dinámica del mercado o en la capacidad de pago de las personas, con ingerencia limitada del Estado. Quien tenía los medios, pagaba. Quien no los tenía, pedía. En todo caso, predominaba el mercado, a pesar de los intentos de articulación desde el Estado desde la década del 30 (Hernández, 2000b).

Durante los años 60, el país entró en la ruta de la planificación para el desarrollo socioeconómico desde el Estado, en el marco de los pactos internacionales establecidos en la alianza para el progreso. En el modelo internacional, la salud sería asumida como una inversión pública, y el sistema de salud debía estar articulado desde el Estado, bien a través del aseguramiento universal o de un servicio único de salud administrado y prestado directa o principalmente por el Estado (OPS/ OMS, 1966). Pero tal propósito fue influido y transformado por el pacto político entre los dos partidos tradicionales que instauró el Frente Nacional, entre 1958 y 1974. Dicho pacto, prolongado de facto hasta 1986, 
recompuso el modelo bipartidista predominante en el sistema político y superó la amenaza populista representada en la experiencia de gobierno militar del General Gustavo Rojas Pinilla entre 1953 y 1957. Al mismo tiempo, la magnitud y el desarrollo del pacto condujeron al fortalecimiento del Estado excluyente, clientelista y patrimonial ista que tanto pesa hoy en el conflicto armado y en la escasa legitimidad del Estado colombiano (Hartlyn, 1993).

Los actores sociopolíticos del campo de la salud también entraron en una lógica patrimonialista. En el momento en que la Ley 12 de 1963 (Colombia, 1963) ordenó desarrollar un sistema único de algún tipo, sobre la base de la ampliación de la asistencia pública, cada cual salió a defender sus fueros. Los trabajadores sindicalizados y los empleados públicos defendieron los servicios de salud por los cuales cotizaban, sin duda mejores que los ofrecidos por la asistencia pública. Los grandes empresarios desconfiaban de la administración pública de sus aportes, a pesar de los beneficios del modelo proteccionista que el Frente Nacional sostenía. Las juntas de beneficencia y la I glesia habían acumulado poder en las regiones a partir de las instituciones de caridad, y no estaban dispuestas a entregar sus bienes y servicios al poder central. Pero también querían los auxilios del Estado. Los médicos se dividieron entre los que defendían su vinculación con las instituciones de seguro social y de asistencia pública, y los que promovían el ejercicio privado de la profesión (Hernández, 1998).

Los habitantes más pobres, tanto del campo como de las ciudades en expansión, pedían hospital y médico para resolver sus problemas, y siempre había un político, liberal o conservador, que prometía el servicio. En ocasiones, el político cumplía con la construcción, como parte de los mecanismos establecidos de intermediación política entre centro y periferia, pero no con el funcionamiento del hospital o del centro de salud. O, también, la población, de todas las clases sociales, utilizaba las prácticas médicas populares e indígenas, más por la desconfianza cultural hacia la medicina occidental que por la ausencia de servicios.

Así las cosas, la articulación del sistema debió sufrir varios embates, a pesar de la formación de los salubristas planificadores que desarrollaron y ocuparon los cargos técnicos del Ministerio de Salud (MS). La “salud pública” se comenzaba a entender como un sector del Estado encargado de la salud de toda la población y no solamente de la atención y la prevención de epidemias. Cuando este grupo de tecnócratas convencidos Ilevó al Congreso la pro- puesta de conformación de un Servicio Único de Salud, en 1972, el proyecto se tachó de comunista. Se asimiló al modelo defendido y desarrollado por el presidente chileno Salvador Allende. El sistema político colombiano, decididamente bipartidista y excluyente, rechazó en el Congreso tal propuesta. Los salubristas se apoyaron entonces en Ia Teoría General de Sistemas para hacer convivir todas las viejas formas de prestación de servicios en un SNS (Quevedo et al., 1990). Mediante facultades especiales al ejecutivo, el cuerpo técnico del MS diseñó el sistema durante los años 70, con la idea de articular "funcionalmente" tres subsectores en todo independientes: el oficial, el de seguridad social y el privado. El "cerebro" sería un gran ministerio técnico, y los "aparatos conectores" serían los subsistemas funcionales de información, infraestructura, recursos humanos, entre otros. La Figura 1 muestra la estructura básica del SNS establecido por el Decreto-Ley 056 de 1974 (Colombia, 1974).

Pero el intento resultó fallido. Cada subsector conservó su autonomía y profundizó la desigualdad. Sin duda, la beneficencia perdió espacio una vez se establecieron los contratos

Figura 1

Estructura del Sistema Nacional de Salud (SN S). Colombia, 1974.

En el contexto de un Estado interventor, planificador, centralista y asistencialista

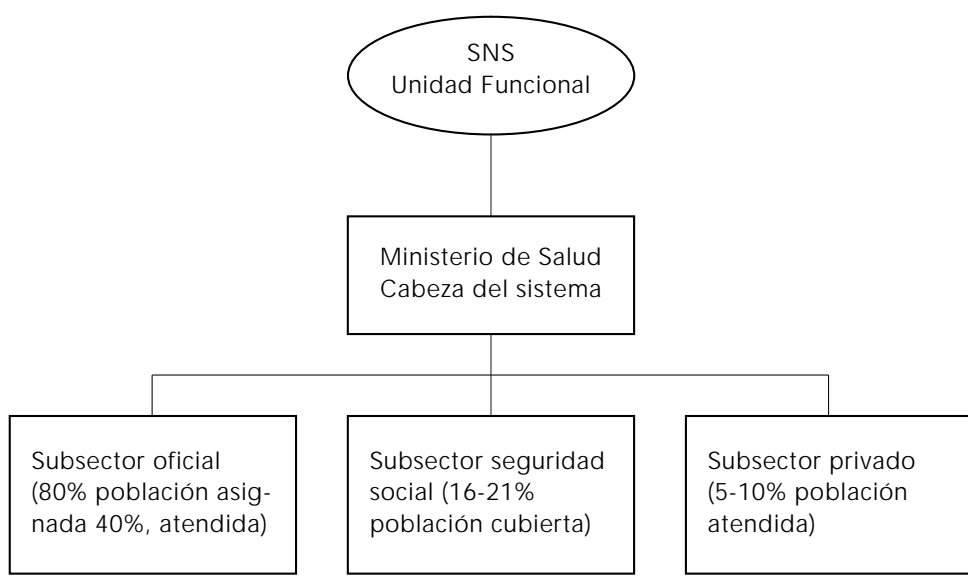

Subsistemas de articulación entre los subsectores (Información, recursos, infraestructura, planeación, etc) 
con el Estado para recibir recursos y ampliar la red destinada a la asistencia pública. Pero la lógica de la caridad siguió primando en este esquema. Además, los contratos fueron varias veces demandados con éxito, por su inconstitucionalidad y porque no había claridad sobre la persona jurídica que vendría a ser cada uno de estos centros y hospitales. Esta indefinición jurídica pesa aún hoy, como parte de la crisis hospitalaria que atraviesa el sistema en la actualidad.

El Instituto Colombiano de Seguros Sociales tuvo su propia reforma en 1977 y se distanció totalmente del control y del esfuerzo de articulación a través del Sistema. Entre tanto, crecían, de manera dispersa y desigual, nuevas cajas de seguros para los empleados de las instituciones públicas. El subsector privado fue creciendo progresivamente a través de los esquemas de prepago, desarrollados por el sector financiero y siempre subvalorados a la hora de definir las coberturas del sistema en su conjunto. Entre tanto, las acciones de control y prevención de enfermedades se articularon a la red pública de centros y hospilares. Así las cosas, en la década de los 80, la expresión "Sistema Nacional de Salud" se comenzó a aplicar sólo a esa red pública y no al conjunto de prestadores de servicios, mientras los demás “subsectores" siguieron su propio rumbo.

Puede decirse, entonces, que el desarrollo del SNS comprobó la fuerza sociopolítica de cada uno de los subsectores que habían crecido en el marco de la lógica del mercado, frente a un Estado clientelista y patrimonialista, al que también convenía la permanencia de estas fracturas de la organización de los servicios de salud. El intento de reforma del sistema, a finales de los años 80, con recursos del Banco Mundial destinados al proyecto de “Consolidación del Sistema Nacional de Salud", sólo terminó en una descentralización del manejo de la red pública de salud hacia los municipios mediante la Ley 10 de 1990 (Colombia, 1990), pero con muchas dificultades administrativas y financieras para su ejecución. Hoy todavía se aprecian los escollos para la descentralización, no sólo técnicos, sino políticos, relacionados con Ias viejas prácticas clientelistas del Estado, o con la simple y franca corrupción incontrolable, o con el conflicto armado cada vez más extendido.

Así las cosas, el SNS lo único que hizo fue legalizar y, por esa vía, legitimar, la inequidad acumulada en el terreno de la asistencia sanitaria. Esto es, atención para ricos, para sectores medios y para pobres, aunque existieran duplicaciones y, a veces, los pudientes utiliza- ran los beneficios de una asistencia pública indiscriminada. Los cálculos más pesimistas sobre la cobertura del Sistema a finales de los ochenta hablaban de $40 \%$ en el subsector oficial, $18 \%$ en la seguridad social, $17 \%$ en práctica privada, especialmente sustentada en el prepago, y un $25 \%$ de población desprotegida. Más que diferencias en cobertura, era claro que estos tres subsectores tenían diferencias en calidad, oportunidad y eficiencia, además de las reconocidas desigualdades geográficas en el acceso (MS, 1994).

La cultura política que sustentaba la fragmentación se inscribía en el derecho a la propiedad, de la cual se derivaban los demás derechos. Quien tenía, obtenía beneficios, con base en su capacidad de pago. Quien no tenía, pedía y obtenía algo, gracias a la caridad pública o privada, siempre precaria y caprichosa. Éste no es el sustento de un Estado Social o del Bienestar como el que se consolidó en algunos países europeos sobre la base de la garantía de los derechos económicos, sociales y culturales consignados en la Declaración de los Derechos Humanos, en 1948. Tampoco se construyó ciudadanía en el desarrollo del SNS, en el sentido de la práctica cotidiana de un sujeto de derechos y deberes en relación con los otros y respecto de un Estado Social de Derecho. En consecuencia, a pesar de que el Estado colombiano suscribió formalmente el Pacto de Derechos Económicos, Sociales y Culturales con la Ley 74 de 1969 (Colombia, 1969), tampoco puede decirse, como afirma Sarmiento (1997), que estos derechos "entraron en vigor el 3 de enero de 1976".

\section{El sistema general de seguridad social en salud: la nueva fragmentación}

La inconformidad social con el SNS se venía expresando en diferentes medios. Al mismo tiempo, durante la década de los 80, se profundizó la crisis de los Estados nacionales en la medida en que ésta fue presionando la nueva expansión de las economías capitalistas, con su terciarización y globalización de los procesos productivos, de distribución y de consumo (Restrepo et al., 1996). Aparecían nuevos actores en el campo de la salud, como las empresas de medicina prepagada, cada vez más fuertes y articuladas a la expansión de la banca internacional. También aparecían nuevas demandas sociales, representadas en movimientos cívicos reivindicativos regionales y locales, protestas urbanas más o menos organizadas, y movimientos étnicos fortalecidos. En la coyuntura de un proceso de desmovilización de varios 
grupos guerrilleros, en especial del M-19, en un nuevo intento de incorporación a la vida civil, las fuerzas políticas tradicionales impulsaron una Asamblea Nacional Constituyente, que culminó en la nueva Constitución Política de 1991 (Asamblea Nacional Constituyente, 1991). En este escenario, se produjo la expresión clara de todas las tendencias que vendrían a aparecer en el proceso de discusión y formulación del nuevo Sistema de Seguridad Social, excepto las de los grupos insurgentes que no entraron en el pacto. Esto es, el Ejército de Liberación Nacional (ELN) y las Fuerzas Armadas Revolucionarias de Colombia (FARC).

En un trabajo previo, se presentó un mapa delas posiciones políticas manifestadas por los actores sociopolíticos nacionales e internacionales que en ese momento se observaron (Hernández, 1998). En la Tabla 1, se presenta una síntesis de las posiciones más importantes registradas en diferentes fuentes, en especial en la recopilación oficial realizada por el MS (1994). Pero el punto central está en reconocer que, en el ambiente sociopolítico de comienzos de la década de los 90, existían en Colombia posiciones que iban desde el neoliberalismo extremo hasta una socialdemocracia amplia, dependiendo del lugar que cada actor ocupaba en el campo de la salud. En el calor de las discusiones en el Congreso, en el seno de los equipos de nuevos tecnócratas de gobierno formados en la nueva economía de la salud, en escenarios más o menos académicos, más o menos políticos, se fraguó el nuevo modelo.

El modelo resultante recogía elementos del debate internacional, con la convicción, en todo caso, de disminuir progresivamente la prestación directa de servicios por parte del Estado, de permitir la concurrencia de actores en el mercado, tanto de prestación como de aseguramiento, y de controlar, al mismo tiempo, las desigualdades producidas por la capacidad de pago, problema reconocido a esa altura en todas partes (Restrepo, 1997). Algunos actores sociopolíticos lograron mantenerse por fuera del nuevo sistema, conscientes de la posibilidad de perder derechos adquiridos, como los empleados del sector de educación pública (FE-

Tabla 1

Las propuestas en el debate sobre la reforma.

\begin{tabular}{|c|c|}
\hline Actor & Características de la propuesta \\
\hline $\begin{array}{l}\text { Departamento Nacional de Planeación, } \\
\text { Ministerio de Hacienda, Ministerio del Trabajo }\end{array}$ & Projecto de Ley 155/92. Fondos privados de pensiones como flexibilización laboral. \\
\hline Instituto de Seguros Sociales & $\begin{array}{l}\text { Conservar Instituto de Seguros Sociales, separar salud de pensiones, aumentar } \\
\text { cotizaciones y mejorar gestión. }\end{array}$ \\
\hline Ministerio de Salud (AD-M 19) & $\begin{array}{l}\text { Proyecto de seguro universal de salud, Seguro Nacional de Salud, administrando } \\
\text { por el Estado. }\end{array}$ \\
\hline Sindicatos & $\begin{array}{l}\text { Incluir salud en la reforma, conservar el ISS, no a fondos privados de pensiones, } \\
\text { ampliar seguridad social e incluir educación, vivenda, empleo, recreación. }\end{array}$ \\
\hline Empresarios & Privatización mediante mecanismo de seguros, no aumentar cotización. \\
\hline Grupo conservador & $\begin{array}{l}\text { Incluir salud en la reforma, separar pensiones de salud, privatizar salud com sistema } \\
\text { de seguros o medicina prepagada. }\end{array}$ \\
\hline Asociación de Empresas de Medicina Prepagada & Privatización de salud com seguros privados, al estilo chileno. \\
\hline $\begin{array}{l}\text { Asociación Colombiana de Facultades } \\
\text { de Medicina (Asmedas) }\end{array}$ & $\begin{array}{l}\text { Conservar Instituto de Seguros Sociales, separar salud de pensiones, mejorar eficiencia, } \\
\text { ampliar coberturas mediante contratación com sector privado. }\end{array}$ \\
\hline $\begin{array}{l}\text { Asociación Colombiana de Facultades } \\
\text { de Medicina (Ascofame) }\end{array}$ & $\begin{array}{l}\text { Mejorar eficiencia del Instituto de Seguros Sociales o acabarlo, ampliar contratación } \\
\text { como prestadores privados. }\end{array}$ \\
\hline Sindicatos de trabajadores de salud & $\begin{array}{l}\text { Ampliar coberturas del subsector oficial e incorporar al Instituto de Seguros Sociales } \\
\text { y al subsector privado en un servicio único. }\end{array}$ \\
\hline Grupos étnicos & Régimen especial según etnias y culturas. \\
\hline Parlamentarios AD-M 19 & Sistema único de aseguramiento. \\
\hline Grupo Hatogrande & Aseguramiento, mercado regulado, público-privado, con subsidio a la demanda. \\
\hline
\end{tabular}

Fuente: MS (1994). 
CODE), los trabajadores de la empresa petrolera estatal (ECOPETROL), los miembros del Congreso de la República con todos sus trabajadores y las fuerzas militares.

El sistema es bastante conocido, dado el interés que ha despertado. En la Tabla 2, se presenta una síntesis de los tres componentes del sistema de seguridad social: pensiones, salud y riesgos profesionales. Pero, en el caso de salud, es necesario hacer una síntesis de su estructura básica para los propósitos de este artículo.

El nuevo sistema de salud parte de la separación entre los servicios individuales y los servicios colectivos en salud, para dejar los primeros en manos del mercado con competencia regulada y, los segundos, a cargo del Estado. El fundamento de dicha separación se encuentra en la teoría neoclásica de los bienes. Desde esta perspectiva, los bienes privados son aquellos que implican un beneficio individual diferenciado, por el cual las personas estarían dispuestas a pagar. Los bienes públicos son sólo aquellos con altas externalidades positivas o negativas, esto es, que afectan o favorecen simultáneamente a muchas personas en algún sentido. Por esta razón, estos servicios deberían ser costeados con recursos públicos, porque nadie estaría dispuesto a pagar por ellos (Banco Mundial, 1987).

En el modelo de la Ley 100 de 1993 (Colombia, 1993), Ios servicios individuales quedan organizados por la lógica de un mercado de aseguramiento, pero con elementos que incorporan aspectos de la propuesta de la competencia administrada o manejada de Enthoven (1988). Los mecanismos más importantes para la regulación de la competencia, en el caso colombiano, que lo hacen tan atractivo en el contexto internacional, son: la obligatoriedad del aseguramiento, la existencia de un fondo único al que confluyen todos los recursos (Fondo de
Solidaridad y Garantía - FSG), el pago de los aseguradores per capita ajustado por riesgos (Unidad de Pago por Capitación) y la exigencia de un plan mínimo de beneficios (Plan Obligatorio de Salud - POS). En la Figura 2, se muestran las relaciones entre los actores del sistema y el lugar que ocupan los mecanismos de regulación en el régimen contributivo. En la pretensión de un aseguramiento universal, se plantea la coexistencia de dos regímenes: contributivo y subsidiado. El primero, con un plan de beneficios mínimo, denominado POS, para quienes pueden cotizar el $12 \%$ del salario, con aportes distribuidos en $8 \%$ a cargo del empleador y $4 \%$ a cargo del trabajador. Los trabajadores independientes cotizan el $12 \%$ de los ingresos declarados. El régimen subsidiado está dirigido a aquellos que demuestran no poder cotizar. Para ellos, se ofrece plan obligatorio subsidiado, hoy con el $70 \%$ o menos de servicios del POS contributivo. Para detectar a los pobres, los municipios deben aplicar una encuesta anual que combina criterios de la Línea de Pobreza (LP) y de Necesidades Básicas Insatisfechas (NBI), lo que constituye un Sistema de Beneficiarios de Subsidios del Estado (SISBEN). El régimen subsidiado es manejado por unos agentes intermediarios denominados Administradoras del Régimen Subsidiado (ARS), quienes contratan con las instituciones prestadoras de servicios (IPS) la atención de los pobres merecedores del subsidio. Queda un grupo de servicios para la atención de enfermedades de alto costo o catastróficas, con una subcuenta especial del FSG. Se esperaba también la reglamentación de algunos “lujos de hotelería” a los que se denomina "planes complementarios", pero esto no se ha realizado. En la transición, se suponía que la población vinculada al antiguo subsector oficial pasaría a afiliarse en alguno de los dos regímenes, al terminar el año 2001.

Tabla 2

La reforma: sistema de seguridad social.

\begin{tabular}{lll}
\hline Pensiones & Salud & Riesgos profesionales \\
\hline $\begin{array}{l}\text { Dos regímenes: } \\
\text { Prima media }\end{array}$ & Dos regímenes: & Modelo de seguro \\
Ahorro individual & Contributivo & Contratado por empleador \\
Solidaridad & Subsidiado & Con poca participación de trabajadores \\
Posterga independentes & Solidaridad & Excluye independentes e informales \\
$\begin{array}{l}\text { Excepciones (militares, } \\
\text { congresistas, educadores, }\end{array}$ & Posterga independientes & \\
petroleros) & Excepciones (militares, & \\
\hline
\end{tabular}


EI FSG está dividido en subcuentas que le permiten manejar diferentes componentes del sistema. La subcuenta de compensación se destina al pago de las empresas promotoras de salud (EPS), con el monto de las Unidad de Pago por Capitación (UPC) que resulten del número de afiliados. De esta forma, las EPS no compiten por los aportes sino por el número de afiliados. La subcuenta de solidaridad se destina a la financiación del régimen subsidiado, se alimenta con el monto correspondiente a un punto porcentual de los aportes de los afiliados al régimen contributivo con más de cuatro salarios mínimos de ingresos. Los recursos de esta subcuenta se suman a los que provienen de la transferencia de recursos de la Nación a los municipios (Ley 60 de 1993 - Colombia, 1993b) y los recursos propios que los departamentos y los municipios dediquen a este fin. La subcuenta de Promoción de la Salud y Prevención de Enfermedades destina el 0,5\% de las UPC recaudadas por las EPS para el pago de acciones preventivas y de promoción. Esta subcuenta recibe algunos recursos adicionales para acciones colectivas manejadas por el Estado. La subcuenta de enfermedades catastróficas financia el costo de las enfermedades crónicas y de alto costo, no incluidas en el POS.

En este sistema, se separan las funciones de "administración del aseguramiento", a cargo de las EPS, de "prestación de servicios", en manos de las IPS. Se espera que las instituciones públicas y privadas confluyan en un ambiente de competencia y que los usuarios puedan elegir, tanto su EPS como su IPS. Se conservaron Ias instituciones más grandes que administraban los seguros obligatorios como EPS públicas (el Seguro Social, Ia Caja Nacional de Previsión, entre otras), y se inició un proceso de autonomización de los centros y hospitales de la red pública de servicios, con la denominación de empresas sociales del Estado (ESE). Estos hospitales deben competir por los usuarios y establecer contratos con los administradores del aseguramimento. En general, el pago de las IPS por parte de las EPS se hace por facturación de servicios realizados, y las tarifas son definidas libremente por los aseguradores, con base en el comportamiento de la oferta y de la demanda.

El Estado se concentra en cuatro funciones principales: (1) "modulación" o "rectoría” del sistema, en concertación con los actores del sistema en el Consejo Nacional de Seguridad Social y los consejos territoriales que se establezcan; (2) subsidio a la demanda de los extremadamente pobres, con el fin de vincularlos al mercado de aseguramiento; (3) vigilancia y control del cumplimiento de las reglas defini-
Figura 2

Mecanismos de regulación.

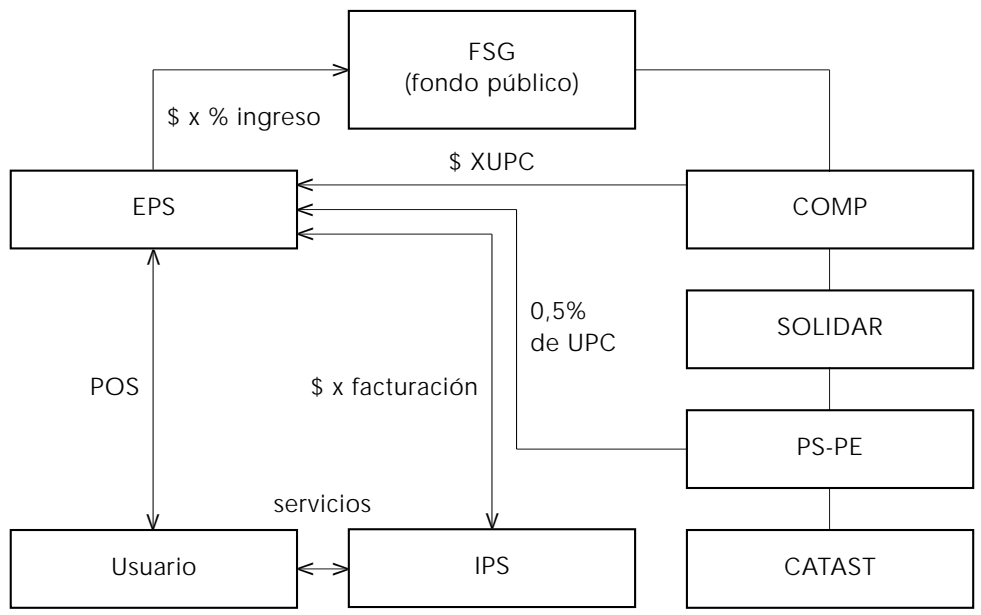

FSG = Fondo de Solidaridad y Garantía; EPS = Empresas promotoras de salud UPC = Unidad de Pago por Capitación; XUPC = pago por UPC;

POS = Plan O bligatorio de Salud; IPS = Instituciones prestadoras de servicios: COMP = Cuenta de compensación; SO LIDAR = Cuenta de solidaridad: PS-PE = Cuenta de promoción de la salud y prevención de enfermedades; CATAST $=$ Cuenta de enfermedades catastróficas.

das; (4) definición y manejo descentralizado de acciones de "salud pública", es decir, aquellas dirigidas al control de problema de salud con altas externalidades, tales como enfermedades de alto impacto, epidemias y desastres.

Seis años después de iniciado el desarrollo de este modelo, la equidad en el acceso a los servicios de salud deja todavía mucho que desear. El primer problema se expresa en el aumento progresivo del gasto en salud, mientras las coberturas siguen dejando mucho que desear. Aunque los cáculos aún son imprecisos, se reconoce un aumento del gasto total en salud que va del orden del 6 o 7\% del Producto Interior Bruto (PIB), en 1990, al 10 o 10,5\% del PIB en 1999 (Málaga et al., 2000). Según el informe de actividades del MS al Congreso de la República de 1999-2000, el 32,8\% de la población se encuentra afiliada al régimen contributivo y el $22,5 \%$, al régi men subsidiado ( $M a ́ l a g a$ et al., 2000). La depuración de la información por parte de la Superintendencia Nacional de

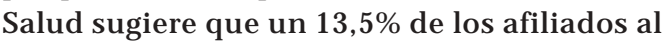
régimen contributivo pueden ser repetidos (Málaga et al., 2000), mientras el régimen subsidiado apenas cubre cerca de $50 \%$ de la población en condiciones de pobreza, según el indicador de NBI (Herrera, 1999). El resto de la población se inscribe, entre un 5 y un $10 \%$, en la 
"medicina prepagada”, o en el régimen de transición al que se ha denominado "vinculado", en el cual los pacientes pagan porcentajes de las tarifas en el momento de consultar la red pública de servicios, según su clasificación en el SISBEN. Queda un porcentaje pequeño, cercano al 3\%, de trabajadores en regímenes especiales. En suma, cerca del $40 \%$ de la población se encuentra en condiciones de vulnerabilidad. Esto es, en esa zona entre la afiliación y la desafiliación social completa de la que habla Castel (1997) en su historia de largo plazo sobre la construcción de lo social.

Lo anterior indica que la meta de universalidad aún no se cumple y es poco probable que se pueda alcanzar en el mediano plazo, dada la caída progresiva del crecimiento económico, la persistencia de la tasa de desempleo por encima del $20 \%$, la política de disminución del gasto público en medio del ajuste fiscal y la tendencia sostenida a la flexibilización laboral (Agudelo, 1999; Hernández, 2000a; Rodríguez, 2000). En estas condiciones, sólo unos pocos podrán afiliarse con base en su relación laboral, mientras aumenta la cantidad de población que se verá obligada a acudir a la competencia por el subsidio estatal o a su capacidad de pago en el momento de enfermar.

Por otra parte, el efecto económico de la intermediación financiera sigue sin una valoración adecuada. Han aumentado el gasto público y el privado en atención médica, pero no han aumentado las coberturas. Esto sugiere un problema de eficiencia que está por explorarse y que apunta a una evaluación seria del papel de los aseguradores y de los intermediarios.

La situación también muestra una incapacidad del sistema para superar las tradicionales desigualdades acumuladas por el sistema anterior. A pesar de los mecanismos de regulación, la relación entre planes de beneficios y mecanismos de financiación ha configurado otra fragmentación que va de la libertad contractual a la caridad, como se muestra en la Tabla 3.

Con base en su capacidad de ingerencia en el Consejo Nacional de Seguridad Social en Salud, las empresas de seguros, hoy convertidas en EPS privadas, han logrado sostener sus antiguos planes de "medicina prepagada”, y se han negado a la reglamentación de los "planes complementarios" que ordena la Ley. De esta forma, un porcentaje pequeño de la población, con altos ingresos, sostiene una medicina privada costosa y con diferencias claras en términos de calidad, oportunidad y accesibilidad.

Estos planes se comportan como un simple seguro privado ligado a la capacidad de pago y con las dos características básicas de cualquier mercado. Esto es, libre elección del usuario y competencia entre oferentes por el precio de las pólizas. Aquí sólo es posible exigir al Estado el cumplimiento de un contrato (derecho contractual) entre consumidores, aseguradores y prestadores. La exclusión que resulta es obvia: quien no tiene, no obtiene.

En segundo término, se encuentra el POS del régimen contributivo. Es claro que la ley establece un plan de beneficios amplio que podría cubrir cerca del $90 \%$ de las patologías propias del perfil epidemiológico del territorio colombiano. Pero aquí se configura sólo el derecho contractual regulado y la exi gibilidad se reduce al cumplimiento de tal contrato. Con frecuencia los usuarios no conocen en detalle el plan de beneficios y sus expectativas están elevadas en el momento de enfermar o percibir el riesgo de muerte; por su parte, los prestadores se ven constreñidos a la letra del contrato a la hora de tomar decisiones clínicas, y los aseguradores insisten en el control de costos. Aún está por hacerse un estudio detallado de los problemas que acarrea esta relación usuarioprestador-asegurador en términos de la equidad en el acceso, la oportunidad y la calidad del servicio. La reciente encuesta realizada por la Defensoría del Pueblo (c. 2000) sobre la calidad percibida por los usuarios es absolutamente insuficiente para aproximarse a la complejidad de esta relación que, en todo caso, se expresa en el aumento sostenido del número de quejas a la Superintendencia de Salud y a la Defensoría del Pueblo, y de tutelas presentadas ante la Corte Constitucional en asuntos de saIud (Corporación Salud y Desarrollo, 1998; Giraldo, 2000).

EI POS subsidiado no ha podido pasar del $70 \%$ de los beneficios del POS contributivo, en sus mejores momentos. Esto implica una desigualdad en la accesibilidad a los servicios, justo en la población que más se encuentra sometida a riesgos de enfermedad y muerte. La presencia de altas tasas de morbi-mortalidad por enfermedades crónicas, degenerativas y por lesiones entre población pobre (Málaga et al., 2000) muestra la presencia de una heterogeneidad estructural, más que una transición epidemiológica, en el caso colombiano. Pero esta situación no parece estar en la mira del actual sistema. Sólo se trata de paquetes de beneficios, costos y recursos disponibles. La deuda del aporte público a la subcuenta de solidaridad del FSG (Chamucero, 1999) presiona hacia abajo las coberturas del régimen subsidiado y la posibilidad de ofrecer, por lo menos, el mismo plan del régimen contributivo. En todo ca- 
Los derechos en el Sistema de Seguridad Social Colombiano.

\begin{tabular}{lllll}
\hline Servicios & Planes de beneficios & Mecanismo & Derecho & Inclusión-Exclusión \\
\hline Individuales & Medicina prepagada & Mercado libre & Contractual & Capacidad de pago \\
& POS contributivo & Mercado regulado & Contractual mínimo & Relación salarial \\
& POS subsidiado & Caridad mediatizada & Contractual limitado & Condición de pobre extremo \\
& Vinculados & Caridad a medias & Caridad limitada & Capacidad de pago (menor) \\
& ATEP & Mercado & Contractual limitado & Relación salarial y empleador \\
Colectivos & PAB & Administración estatal & Ciudadano & Altas externalidades
\end{tabular}

ATEP = Accidentes de Trabajo y Enfermedad Profesional; PAB = Plan de Atención Básica; POS = Plan O bligatorio de Salud.

so, la población beneficiaria ha entrado en la lógica de la competencia por la limosna estatal. En este terreno, no es posible configurar el sentido de derecho ciudadano. A duras penas, los usuarios más avesados descubren que existe un contrato de obligatorio cumplimiento por parte de una ARS y que tienem derecho a reclamar su cumplimiento.

Entre tanto, los que continúan en el régimen de vinculados están accediendo a un sistema de caridad a medias que en todo caso ofrece el obstáculo de la capacidad de pago. El sistema de riesgos profesi onales se ubica en un esquema paralelo de seguro colectivo definido por el empleador, contractual y excluyente, a pesar de la existencia de mecanismos de participación de los trabajadores definidos en la Ley y sus decretos reglamentarios (Luna, sometido).

El único plan de beneficios que parece no tener una configuración contractual es el Plan de Atención Básica (PAB), porque supuestamente está a cargo del Estado, es gratuito y de acceso universal. El problema está en que cada vez más se reduce a acciones estrictamente colectivas, con altas externalidades y de alta prioridad social y económica. El Estado trata de ubicar las acciones preventivas en el POS, para que sean responsabilidad de las EPS y ARS, mientras éstas se resisten por la dificultad de definir cuándo estas acciones preventivas desbordan el servicio individual y complican su facturación por parte de las IPS. De allí la baja ejecución de la subcuenta de promoción y prevención del FSG (Hernández, 2000a). La desterritorialización propia del mercado en los servicios individuales ha ocasionado también un abandono de responsabilidades institucionales de una serie de programas de control de enfermedades prioritarias que nadie está dispues- to a asumir. No de otra forma se explican los aumentos en las tasas de enfermedades como la malaria, el dengue hemorrágico, la tubercuIosis, la sífilis congénita, entre otras (Franco, 1998; Málaga, 2000).

\section{Conclusión}

Con todo, es posible afirmar que, en Colombia, muy a pesar del flamante primer lugar en "equidad financiera" en el mundo, el sistema de seguridad social en salud no ha logrado resolver los problemas de equidad acumulados en el sistema anterior, el cual legalizaba las desigualdades ligadas a la capacidad de pago de las personas. El sistema producto de la reforma de 1993 pretende ser universal, equitativo, eficiente y de mejor calidad, pero aún no es posible hablar de éxitos claros en ninguno de estos objetivos. La opción colectiva adoptada en el seno de un sistema político todavía excluyente y en el marco de una cultura política individualista que no parece haberse modificado en la última década afianza la idea del derecho a la salud como un problema de acceso individual a servicios de atención médica, en virtud de contratos individuales sostenidos en relaciones de mercado, más o menos reguladas, o en francas estrategias de caridad pública consumida individualmente pero con intermediarios de dudosa eficiencia.

En estas condiciones, sería atrevido afirmar que en Colombia avanzamos hacia la consolidación de un derecho a la asistencia sanitaria de tipo universal e incluyente. Por el contrario, un modelo con complejos mecanismos de regulación parece ocultar un problema de fondo que no parece resuel to: la idea de la salud co- 
mo un servicio de consumo individual que puede universalizarse por la vía del mercado. Pero esta convicción tiende a sostener desigualdades injustas y superables. Nos encontramos, entonces, frente a un reto doble: la superación de las obstáculos que se derivan de tal convicción y la construcción de nuevas formas de organización de los servicios y de los recursos disponibles para garantizar el derecho universal a la salud.

\section{Referencias}

AGUDELO, C., 1999. Sistema de salud y plan de desarrollo: Cobertura y equidad. Revista de Salud Pública, 1:117-127.

ASAMBLEA NACIONAL CONSTITUYENTE, 1991. Constitución Política de Colombia 1991. Gaceta Constitucional, No 116. Bogotá: Asamblea Nacional Constituyente.

BANCO MUNDIAL, 1987. El financiamiento de los servicios de salud en los países en desarrollo. Una agenda para la reforma. Boletín dela Oficina Sanitaria Panamericana, 103:695-709.

CASTEL, R., 1997. Las Metamorfosis dela Cuestión Social. Una Crónica del Salariado. Buenos Aires: Paidós.

CESPEDES, J. E.; JARAMILLO, I.; MARTINEZ, R.; OLAYA, S.; REYNALES, J.; URIBE, C.; CASTAÑO, R. A.; GARZON, E.; ALMEIDA, C.; TRAVASSOS, C.; BASTO, F. \& ANGARITA, J., 2000. Efectos de la reforma de la seguridad social en salud en Colombia sobre la equidad en el acceso y la utilización de servicios de salud. Revista de Salud Pública, 2:145-164.

CHAMUCERO, R., 1999. Boletín informativo del representante de los trabajadores (CUT). Consejo Nacional de Seguridad Social en Salud, 4:5-6.

COLOMBIA, 1963. Ley 12 , de 17 mayo. Por la cual se ordena al Gobierno la elaboración del plan hospitalario nacional y se dictan otras disposiciones. In: História de las Leyes, tomo XXI, pp. 406-408, Bogotá: Imprenta Nacional.

COLOMBIA, 1969. Ley no 74, 26 de diciembre, Por la Cual se Ratifica el Protocolo Facultativo del Pacto de Derechos Civiles y Políticos de la Asamblea General de las Naciones Unidas de 1966. Bogotá: Policopiado.

COLOMBIA, 1974. Decreto-Ley no 056, Por el Cual se Sustituye en Decreto-Ley no 654 de 1974 y se Dictan otras Disposiciones. Bogotá: Policopiado.

COLOMBIA, 1990. Ley 10. Bogotá: Congreso de la República.

COLOMBIA, 1993b. Ley 60. Bogotá: Congreso de la República.
COLOMBIA, 1993a. Ley № 100, del 23 de diciembre de 1993. Crea el Sistema de Seguridad Social Integral y se dictan otras disposiciones. Bogotá: Diario Oficial del Congreso de la República de Colombia., Año CXXIX, no 41148, p. 40.

CORPORACION SALUD Y DESARROLLO, 1988. Fortalecimiento dela Promoción dela Salud en el Sistema de Seguridad Social en Santafé de Bogotá. Informe final de asesoría a la Secretaría Distrital de Salud de Santafé de Bogotá. Bogotá: Secretaría Distrital de Salud de Santafé de Bogotá. (mimeo.)

DEFENSORIA DEL PUEBLO, c. 2000. Primera Encuesta Nacional deCalidad Percibida por los Usuarios. s.I.: Defensoría Delegada para la Salud y la Seguriad Social, Defensoría del Pueblo.

ENTHOVEN, A., 1988. Managed competition of alternative delivery systems. Journal of Health Politics, Policy and Law, 13:305-321.

ESLAVA, J. C., 1999. Seguridad social en salud: Reforma a la reforma. Revista deSalud Pública, 1:105-116.

FRANCO, S., 1998. Hacia una política nacional de salud pública. Cartilla de Seguridad Social, 5:71-82.

FRENK, J. \& LONDOÑO, J. L., 1997. Pluralismo estructurado: Hacia un modelo innovador para la reforma de los sistemas de salud en América Latina. Salud y Gerencia, 15:6-28.

GIRALDO, J. C., 2000. Ejercicios del Derecho a la Salud en Colombia. Bogotá: Defensoría del Pueblo.

HARTLYN, J., 1993. La Política del Régimen de Coalición. La Experiencia del Frente Nacional de CoIombia. Bogotá: Tercer Mundo, Centro de Estudios Internacionales, Universidad de los Andes.

HERNANDEZ, M., 1998. El proceso sociopolítico de las reformas sanitarias en Colombia. Cartilla de Seguridad Social, 5:21-43.

HERNANDEZ, M., 2000a. El derecho a la salud en CoIombia: Obstáculos estructurales para su realización. Revista de Salud Pública, 2:121-144.

HERNANDEZ, M., 2000b. La Fractura Originaria en la Organización de los Servicios de Salud en Colombia. 1910-1946. Tesis de Magíster, Bogotá: Universidad Nacional de Colombia. 
HERNANDEZ, M., 2000c. El enfoque sociopolítico para el análisis de las reformas sanitarias en América Latina. In: VIII Congreso Latinoamericano de Medicina Social y XI Congreso de la Asociación Internacional de Politicas de Salud, Memórias de Ios Congresos, CD-ROM, La Habana: Asociación Internacional de Políticas de Salud.

HERRERA, V., 1999. Régimen subsidiado de salud: Evolución y problemática. Revista deSalud Pública, 1:193-211.

LUNA, J. E., (sometido). Alcances y limitaciones en el desarrollo del Sistema General de Riesgos Profesionales. Revista dela Contraloría General dela Nación.

MALAGA, H.; LATORRE, M. C.; CARDENAS, J.; MONTIEL, H.; SAM PSON, C.; TABORDA, M. C.; GRANADOS, R. \& PASTOR, D., 2000. Equidad y reforma en salud en Colombia. Revista deSalud Pública, 2:193-219.

MS (Ministerio de Salud), 1994. La Reforma a la Seguridad Social en Salud. Antecedentes y Resultados. Bogotá: MS.

OMS (Organización Mundial de la Salud), 2000. Informe sobrela Salud en el Mundo, 2000. Mejorar el Desempeño de los Sistemas de Salud. Geneva: OMS.

OPS (Organización Panamericana de la Salud)/ OMS (Organización Mundial de la Salud), 1966. Administración de Servicios de Atención Médica. Nuevos Elementos para la Formulación de una Política Continental. Publicación Científica 129. Washington, DC: OPS/OMS.
QUEVEDO, E.; HERNANDEZ, M.; MIRANDA, N.; MARIÑO, C.; CARDENAS, H. \& WIESNER, C., 1990. La Salud en Colombia. Análisis Sociohistórico. Bogotá: Estudio Sectorial de Salud, Departamento Nacional de Planeación, Ministerio de Salud.

RESTREPO, M., 1997. La Reforma a la Seguridad Social en Salud de Colombia y la Teoría de la Competencia Regulada. Santiago de Chile: Comisión Económica para América Latina y el Caribe, Naciones Unidas.

RESTREPO, M., 2000. Elementos para una doctrina del derecho a la salud en Colombia. In: Ejercicios del Derecho a la Salud en Colombia (J. C. Giraldo, org.), pp. 51-121, Bogotá: Defensoría del Pueblo.

RESTREPO, D.; PALACIO, G.; NOVOA, E. \& GONZALEZ, J. I., 1996. Globalización y Estado Nación. Bogotá: Escuela Superior de Administración Pública.

RODRIGUEZ, O., 2000. Financiarización y flexibilización laboral en el plan de desarrollo “cambio para construir la paz". Revista de Salud Pública, 1:97-104.

SARMIENTO, L., 1997. De los derechos sociales, económicos y culturales. In: Constitución Política de Colombia, Comentada por la Comisión Colombiana de Juristas. Título II: De los Derechos, las Garantías y los Deberes (M. Barreto \& L. Sarmiento, org.), pp. 167-172, Bogotá: Comisión Colombiana de Juristas.

Recebido el 9 de febrero de 2001

Versión final presentada el 9 de febrero de 2001

Aprobado el 3 de octubre de 2001 